\title{
Pancreatic juice outflow in pancreatojejunostomy monitoring with the inter-anastomosis drainage tube; a retrospective observational study
}

\author{
Osamu Shimomura \\ University of Tsukuba \\ Tatsuya Oda ( $\square$ tatoda@md.tukuba.ac.jp ) \\ University of Tsukuba \\ Yoshihiro Miyazaki \\ University of Tsukuba \\ Kinji Furuya \\ University of Tsukuba \\ Manami Doi \\ University of Tsukuba \\ Kazuhiro Takahashi \\ University of Tsukuba \\ Jaejeong Kim \\ University of Tsukuba \\ Shoko Moue \\ University of Tsukuba \\ Yohei Owada \\ University of Tsukuba \\ Koichi Ogawa \\ University of Tsukuba \\ Yusuke Ohara \\ University of Tsukuba \\ Yoshimasa Akashi \\ University of Tsukuba \\ Tsuyoshi Enomoto \\ University of Tsukuba \\ Shinji Hashimoto \\ University of Tsukuba
}


Keywords: Blumgart, Drainage, Pancreatic Fistula, Pancreatoduodenectomy, Pancreatojejunostomy Posted Date: January 27th, 2022

DOI: https://doi.org/10.21203/rs.3.rs-1236597/v1

License: (c) (1) This work is licensed under a Creative Commons Attribution 4.0 International License. Read Full License 


\section{Abstract}

Background:

Pancreatic fistula remains the biggest problem in pancreatic surgery. We have previously reported a new pancreatojejunostomy method using an inter-anastomosis drainage suction tube (IAD) with Blumgart anastomosis for drainage of the pancreatic juice leaking from the branched pancreatic ducts. This study aimed to evaluate the postoperative outcomes of our novel method, in pancreatojejunostomy and investigate the nature of the inter-anastomosis space between jejunal wall and pancreas parenchyma.

\section{Methods:}

This retrospectively study consist of 282 pancreatoduodenectomy cases, including 86 reconstructions via the Blumgart method plus IAD ( $B+I A D$ group) and 196 cases reconstructed using the Blumgart method alone (B group). Post-operative outcomes and the amylase value and the volume of the drainage fluids were compared between the two groups. The IAD tube was placed to collect amylase-rich fluid from the inter-anastomosis space during operative procedure between the jejunal wall and pancreatic stump.

Results:

The daily IAD drainage volume and the amylase level was significantly higher in patients with a soft pancreas (vs hard pancreas; 16.5 vs $10.0 \mathrm{~mL} /$ day, $p=0.012 ; 90,900$ vs $1,634 \mathrm{IU} / \mathrm{L}, p<0.001$, respectively). The mean amylase value of IAD collection in 86 cases of B+IAD group was $63,100 \mathrm{IU} / \mathrm{L}$. The incidence of clinically relevant pancreatic fistula grade $B$ and C $(23.2 \%$ vs. $23.0 \%, p=0.55)$ and the hospital stay was similar between the groups (median 17 vs. 18 days, $p=0.55$ ). In 176 patients with soft pancreas, the incidence of pancreatic fistula grade $B$ and $C(33.3 \%$ vs. $35.3 \%, p=0.67)$ and the hospital stay was also similar between the groups (median 22.5 vs. 21 days, $p=0.81$ ).

Conclusions:

Positive effect of the IAD method observed in the pilot cases was not reproduced in the current study. IAD tube objectively demonstrated the existence of amylase rich discharge at the anastomosis site, and countermeasures to eliminate this liquid are highly desired for preventing pancreatic fistula, especially in patients with soft pancreatic texture.

Trial registration: Retrospectively registered

\section{Background}

Pancreatic fistula (PF) is still a major issue after pancreaticoduodenectomy (PD) and it is associated with a high mortality rate even with recent advances in surgical procedures and postoperative management ${ }^{1-}$

3 . The rate of clinically relevant postoperative PF (POPF) was higher than $10 \%$ in previous studies ${ }^{2,4}$, and it is an unsolved problem in the field of pancreatic surgery. 
Recent improvements in the surgical technique - Blumgart anastomosis - proposed by Blumgart et al., enables a tight adaptation between the jejunum wall and fragile pancreatic parenchyma, protecting the thin pancreatic coat with jejunal serosa ${ }^{5}$. Several modifications in suture methods have been reported ${ }^{6-}$ 8 , and this anastomosis is now widely accepted. However, Blumgart anastomosis did not significantly reduce clinically relevant POPF compared with conventional interrupted sutures based on a randomized controlled study reported by Hirono et al. ${ }^{9}$.

A soft pancreatic consistency with the normal ability to produce pancreatic juice is a well-known risk factor for POPF after PD ${ }^{4,10}$. For a soft pancreatic texture, countermeasures are required to prevent pancreatic juice efflux from both the main pancreatic duct (MPD) and branch duct. The main causes of PF are divided into two categories. The first is leakage from the anastomosis of the MPD and mucosal membrane, and the second is leakage from small openings of branch pancreatic ducts in the parenchymal stump. Although Blumgart anastomosis enables tight adhesion between the jejunal wall and pancreatic stump, it may not be enough to reduce leakage from the branch duct of the pancreatic parenchyma. Undesired outflow of pancreatic juice initially collects in the inter-anastomosis space between the pancreatic stump and jejunum serosal wall. Subsequently, the juice flows out from the interanastomosis space into the peritoneal cavity, leading to a long-standing PF. Invagination and dunkingtype reconstruction, the conventional approaches for preventing leakage from the pancreatic stump have been developed, in which the pancreatic stump invaginates into one large jejunal or gastric hole. The drawback of these methods is that activated digestive juice caused by a mixture of pancreatic juice, bile juice, and intestinal flora could induce severe PF.

Once a PF has developed, the healing of the PF requires the successful closure of the MPD and jejunal mucosa anastomosis and/or stopping the outflow of pancreatic juice from the branch duct. Evidence suggests that creating a spontaneous drainage route to the internal jejunal lumen is a key factor in the healing process ${ }^{11,12}$. These findings indicate that the creation of a drainage route between the interanastomosis space and the jejunal lumen at the time of reconstruction might collect the effused juice and prevent PF.

Here, we present the postoperative outcomes of our strategy using a novel add-on method for pancreatojejunostomy. Our method involves the placement of a single-suction drainage tube into the space between the pancreatic stump and jejunum wall, namely the inter-anastomosis drainage (IAD) tube. No methods have been developed to provide information about the leakage of pancreatic juice from the stump. Our method highlights the importance of an undiscovered inter-anastomosis space in pancreatojejunostomy.

\section{Methods}

\section{Patient background and data collection:}


This is a retrospective study including 282 patients who underwent PD with Child-type reconstruction using modified Blumgart-type pancreatojejunostomy ${ }^{7}$ at a single institution in Japan (Department of Gastrointestinal and Hepato-biliary-pancreatic Surgery, University of Tsukuba Hospital) from July 2010 to June 2020. Among the 282 patients, 86 underwent Blumgart with IAD ( $B+I A D$ group), and 196 patients only underwent the Blumgart method (B group). B+IAD was performed between January 2014 and February 2018, and the Blumgart-only (B group) procedure was performed before and after this period. Clinical data and pre- and postoperative data were reviewed from the medical records and analyzed retrospectively. A subgroup analysis was performed for patients with a soft pancreatic texture. All patient data sets were collected after approval by the institutional review board of Tsukuba University Hospital (R01-030).

\section{Outcome assessment:}

POPF was diagnosed according to the classification of the International Study Group on Pancreatic Fistula (ISGPF) ${ }^{13}$. Clinically relevant POPF was defined as grade B or C. Grade B required a change in the postoperative management; drains were either left in place for more than 3 weeks or replaced by endoscopic or percutaneous procedures. Grade $\mathrm{C}$ required reoperation or led to single or multiple organ failure and/or mortality attributable to $\mathrm{PF}^{13}$. The status of the pancreatic parenchyma (soft or hard pancreas) was determined by subjective palpation of the operator. The size of the MPD was measured at the presumed surgical transection line on preoperative contrast-enhanced computed tomography.

\section{Operative Procedure:}

\section{Pancreatic transections:}

Pancreatic transections were performed using a sharp scalpel, and any notable bleeding and recognizable openings of small branches of the pancreatic ducts were ligated with 5-0 monofilament stitches. Hemostasis of small hemorrhages was achieved using electrocautery.

\section{Placement of the inter-anastomosis drainage tube:}

After completion of the duct-to-mucosa anastomosis, the IAD tube $(n=86)$ was placed in the interspace between the jejunal wall and pancreatic parenchyma (Fig. 1a). This procedure was designed to extract undesirable pancreatic juice from the inter-anastomotic space using an active suction system. Briefly, an IAD tube (10 Fr. The BLAKE Silicone Drains-Hubless, Ethicon, code 2226, NJ, USA) was positioned such that the short-channel-suction part was located in the inter-anastomosis space between the pancreas and jejunum (Fig. 1b), the middle extension part was located in the inner lumen of the jejunum, and the distal end protruded from the end of the jejunal limb, which is commonly referred to as Witzel's end. The channel part of the tube was originally in the shape of a " + " sign with 4 flaps, which were arranged into a thin and flat "I" shape by removing the anterior and posterior flaps, and the length was adjusted to the 
width of the pancreatic parenchyma (usually $2.5-3.5 \mathrm{~cm}$ ). A water-tight seal between the active IAD tube and jejunal wall was used to block the reverse flow of the contaminated intestinal juice into the interanastomotic space. For this purpose, the extra-abdominal end of the tube was cut diagonally, passing the sharp end through a small jejunal hole to ensure a tight seal. After creating passive IAD holes or after the placement of the active IAD tube, three ties using the Blumgart sutures were made firmly at the anterior serosal wall of the jejunum, and the IAD tube was completely enveloped by the jejunal walls (Fig. 1C). Reinforcing 4-0 PDS-II sutures were occasionally added to both edges. At the end of the surgery, two prophylactic peritoneal drainage tubes were placed at the superior and inferior sites of the pancreatojejunostomy, and the active IAD tube was connected to a low-pressure, continuous suction device (100 mL Bulb Suction Reservoir, Ethicon, code 2160, NJ, USA). All procedures during PD were performed by or under the direction of three surgeons (O.S., S.H., and T.O.).

\section{Postoperative management:}

The fluid amylase levels from the peritoneal drainage tubes were monitored on postoperative day (POD) 1,3 , and 5. On POD 4-5, the tubes were removed for patients with ISGPF grade ${ }^{14}$ None or BL (biochemical leakage) and were replaced with new 18-22-Fr silicon tubes (Fuji Systems Co., Tokyo, Japan) using interventional radiographic techniques for patients with possible ISGPF grade B or C. In 17 patients in the active IAD group, the volumes drained by the active IAD tube were measured daily, and the amylase levels were measured once on POD 1-3. The nasogastric tubes were removed on POD 1 and liquid oral intake began on POD 1, followed by the administration of a liquid diet beginning on POD 2-3 and solid food beginning on POD 4-7.

\section{Statistical analysis:}

Differences between the two groups (i.e., group B vs group B+IAD) were statistically analyzed using SPSS version 26 (IBM, Armonk, NY, USA). Categorical variables were analyzed using the chi-squared test or Fisher's exact test when $20 \%$ of the expected frequencies were less than or equal to 5 . Continuous variables are presented as both the mean (SD) and median (range) and were analyzed using the Student's $t$-test and Mann-Whitney $U$ test for normally distributed and non-normally distributed variables, respectively. All $p$-values from two-tailed tests were considered statistically significant at $p<0.05$.

\section{Results}

\section{Demographics:}

A total of 282 patients underwent PD during the study period, including 86 patients in the B+IAD group and 196 patients in the B group. The demographics and summary of clinical outcomes are shown in Table 1. There were no significant differences in preoperative factors such as age, sex, diagnosis, prealbumin value, diabetes, preoperative biliary stenting, and physical status (American Society of Anesthesiologists). The operation time was significantly longer in the B+IAD group than in the B group; 
however, there were no significant differences in blood loss and the rate of required portal vein reconstruction. Among the 282 patients, 176 had a soft pancreatic texture. A summary limited to the soft pancreatic texture is shown in Table 2; the patient characteristics did not change. The operating time was also significantly longer in the B+IAD group ( $507 \mathrm{vs.} 458 \mathrm{~min}$ ) for those with a soft pancreas.

\section{The incidence of pancreatic fistula:}

Among the 282 patients with hard and soft pancreatic textures, PF grade B and C occurred in $19(22 \%)$ and $1(1.2 \%)$ patient(s), respectively, in group $B+I A D$ and in $41(21.0 \%)$ and $4(2.0 \%)$ patients, respectively, in group B. Clinically relevant POPF was observed in $20(23.2 \%)$ and $45(23.0 \%)$ patients in the B+IAD and B groups, respectively ( $p=0.546)$. Surgical complications diagnosed as greater than Clavien-Dindo $3 a$ occurred in $25(29.1 \%)$ and $56(28.6 \%)$ patients in the B+IAD and B groups, respectively ( $p>0.99)$. No postoperative mortality was observed in any group. The median postoperative hospital stay (POHS) was 17 and 18 days in the $B+I A D$ and $B$ groups, respectively $(p=0.545)$ (Table 1$)$.

Among the 176 patients with a soft pancreas, 57 underwent $B+I A D$ and 119 patients underwent B-only. The data are shown in Table 2. PF grades B and C occurred in 16 (28.1\%) and 3 (5.3\%) patients, respectively, in the B+IAD group and in $38(31.9 \%)$ and 4 (3.4\%) patients, respectively in the B group $(p=0.67)$. Surgical complications diagnosed as greater than Clavien-Dindo 3a occurred in $24(42.1 \%)$ and $51(42.9 \%)$ patients in the $B+I A D$ and $B$ groups, respectively $(p=0.925)$. The median POHS was 22.5 and 21 days in the $B+\mid A D$ and $B$ groups, respectively $(p=0.81)$.

\section{The effect of the inter-anastomosis drainage tube:}

The maximum amount of IAD drainage fluid for both the soft and hard pancreas is shown in Table 3. Additionally, the amylase value and fluid volume of each patient is summarized in Figure 2. In 86 patients ( $B+I A D$ group), the median maximum volume of drainage fluid was $14 \mathrm{~mL} /$ day (range $0-240 \mathrm{~mL}$ ). The daily maximum drainage was significantly higher in patients with a soft pancreas than in those with a hard pancreas ( $16.5 \mathrm{~mL} /$ day vs $10.0 \mathrm{~mL} /$ day; $p=0.012)$. The amylase level in the IAD drainage fluid was significantly higher in patients with a soft pancreas than in those with a hard pancreas (median 90,900 IU/L vs $1,634 \mathrm{IU} / \mathrm{L} ; p<0.0001)$.

\section{Discussion}

This study examined the effect of our novel concept of indwelling an IAD suction tube to prevent POPF during PD reconstruction. This method is expected to reduce undesired pancreatic juice from the branched pancreatic duct and duct-to-mucosa anastomosis and compensate for the shortcomings of Blumgart-type pancreatojejunostomy. This method successfully collected notably amylase-rich fluids in 
the inter-anastomosis space. However, we could not identify meaningful improvements in terms of the incidence of clinically relevant PF and POHS duration.

Our IAD tube method showed, for the first time, the existence of a substantial volume (maximum of 14.0 $\mathrm{mL} /$ day [median] in total 86 patients from the $B+I A D$ group and $16.5 \mathrm{~mL} /$ day in patients with a soft pancreas, Table 3) of undesirable fluid in the interspace between the jejunal wall and pancreatic parenchyma that contained markedly high amylase levels (median value: $90,900 \mathrm{IU} / \mathrm{L}$ in patients with a soft pancreas). This undesired fluid could be considered the origin of PF. However, it is difficult to ascertain whether fluid effluxed from the branch duct was a minor leakage from the duct-to-mucosa anastomosis. From this result, we suggest that the soft pancreas produced a high amount of amylaserich pancreatic juice and the operator's subjective palpation during the procedure regarding whether the pancreas was soft or hard seems correct.

Although the IAD tube successfully collected fluid with notably high amylase levels at the interanastomosis space in Blumgart-type pancreatojejunostomy, we could not identify any meaningful improvements in clinically relevant PF. PF grades B and C occurred in $28.1 \%$ and $5.3 \%$ of patients, respectively, in the $B+I A D$ group and in $31.9 \%$ and $3.4 \%$ of patients, respectively, in the $B$ group. Our initial report on 17 patients using this novel IAD tube method revealed a significant reduction in the incidence rate of $\mathrm{PF}$ grade $\mathrm{B}$ or $\mathrm{C}$ (the incidence rate was $5.9 \%$; 1 patient out of 17 patients) ${ }^{15}$. The IAD tube functions effectively when the pancreatic cut margin is wide and the MPD is located on the dorsal side (Fig. 3a). Conversely, when the surface area of the pancreatic stump is small or the MPD is located ventrally (Fig. $3 b, c)$, the insertion of IAD tube is troublesome and the result is undesirable.

The reason why the IAD tube method did not improve after the first report may be due to three reasons. The first reason is the technical difficulty in the placement of the IAD tube at the inter-anastomosis space between the jejunal wall and the pancreatic parenchyma, especially in cases including a thin pancreas stump. For the IAD tube to exert a pronounced effect in order to protect leakage from the branch duct, the tube needs to be placed in a water-tight-seal manner. This water-tight seal was made using 2 or 3 Blumgart suture knots at the jejunal surface, while embedding the IAD tube above the duct-to-mucosa anastomosis ${ }^{15}$ (Fig. 1b, c). Moreover, this is occasionally a highly challenging problem especially in the case of a small pancreatic cross-section. When the above side of pancreatic stump is thin and same as the width of IAD tube, the tube placement with a water-tight seal is theoretically impossible. In fact, the 17 patients reported in our first report ${ }^{15}$ were operated by staff surgeons engaged in pancreatic surgery. This suture technique, creating a water-seal adhesion, is technically difficult for trainees. In fact, the operating time was longer in the B+IAD group than the $B$ group in the soft pancreas cohort. This may reflect the reconstruction required more times to prudent suture techniques.

Second, the location of the MPD in the stump was important for effective collection. When the MPD was located on the ventral side of the stump, it was quite difficult to place the IAD tube above the duct-tomucosa anastomosis similar to that in patients with thin parenchyma. Moreover, the fluid that leaks below the anastomosis might not be collected effectively by the IAD tube because the IAD tube was only 
placed above. This might also indicate that the IAD tube could not entirely collect the undesired fluid discharged from both the pancreatic stump and the incomplete duct-to-mucosa anastomosis.

The third reason was that the IAD tube placement may prevent tissue adhesion, which is important for the cure of PF. Though IAD successfully collected the amylase rich fluids, foreign substances may delay selfhealing. The median maximum amount of IAD tube collection was $14.0 \mathrm{~mL} /$ day and more than 100 $\mathrm{mL} /$ day was recorded in some patients. This observation may suggest that the collections from the IAD tube included not only pancreatic juice but also enteric fluids that contained abundant enterobacteria. Though IAD tube placement may trigger bacterial infection around the pancreatojejunostomy, we did not experience any fatal infectious complications during the study period.

To overcome the technical difficulties associated with IAD tube placement, a simpler and more reasonable method is required to allow the pancreatic juice to drain into the jejunum. Recent studies have suggested that the healing of PF requires the amylase-rich fluid to drain spontaneously into the jejunal lumen ${ }^{12}$. To achieve spontaneous drainage at an early stage after PD, the intestinal internal pressure needs to be kept low by the placement of an enteric drainage tube near the pancreatojejunostomy. High intestinal internal pressure may induce the outflow of enteric juice and bacterial infection, which activates proteases. Subsequently, the intentional drainage route in the jejunal wall may enable the fluid to spontaneously drain into the jejunum from the early postoperative period without the placement of an IAD tube. To accomplish this, two key factors, tight adhesion between the jejunal wall and pancreatic stump and low pressure inside the jejunum, are required.

In the present study, the incidence of clinically relevant PF (grade B or C) was $23 \%$ in the total cohort and $34 \%$ in the soft pancreas cohort. These values were relatively high compared to other studies on Blumgart-type anastomosis ${ }^{6,9}$. However, POHS did not change significantly compared to these studies ${ }^{6}$, 8, 16. In fact, we achieved 17 days of POHS after PD without mortality. According to the ISGPF grading system ${ }^{13}$, PF grade $B$ requires a change in the postoperative management; thus, drains were either left in place for more than 3 weeks or replaced by endoscopic or percutaneous procedures. We generally performed an early assessment of the obstruction of drainage tubes on POD 3 or 4 and replacement of tubes under radioscopy if the drainage was not effective. Patients who underwent drain tube replacement or for whom the drainage site was changed under radioscopy were judged to have PF grade B. These facts may be responsible for the increased number of patients diagnosed with PF grade B in our study. Even if a patient experiences clinically relevant PF, the duration of POHS is important.

This study has some limitations. First, this is a retrospective study from a single institute, i.e., a teaching hospital. The sample size was limited, and each procedure was performed by several surgeons, including trainees. All procedures were guided by three staff surgeons; however, maintaining reproducibility was not easy due to the technical difficulty of IAD tube insertion. Second, the judgement of soft or hard pancreatic texture was made subjectively by the surgeons. Potential selection bias is difficult to eliminate, and a precise method to assess pancreas status is warranted. Third, pancreatic exocrine secretion depends on the size of remnant pancreas, acinar cell function, and number of detailed branch ducts in the stump. 
Patients with low exocrine potential may not require this IAD method. Therefore, further studies are necessary to clarify the relationship between remnant pancreatic function and the effect of IAD tube insertion.

\section{Conclusions}

This series highlights the nature of the space between the jejunal wall and pancreatic parenchyma in Blumgart type pancreatojejunostomy using IAD tube drainage. Markedly high amylase-rich fluids were observed in patients with a soft pancreas using IAD suction tube drainage, and these collections may be essential for the development of PF. However, IAD tube method utilized with Blumgart anastomosis did not improve the incidence of clinically relevant PF. For a safer PD with Blumgart pancreatojejunostomy, additional improvements are required to overcome the pancreatic juice leakage in the inter-anastomosis space.

\section{Abbreviations}

IAD: inter-anastomosis drainage

PF: pancreatic fistula

PD: pancreatoduodenectomy

POPF: post-operative pancreatic fistula

MPD: main pancreatic duct

B: Blumgart type anastomosis

ISGPF: International Study Group on Pancreatic Fistula

POHS: post-operative hospital stay

\section{Declarations}

\section{Availability of data and materials}

All data generated or analysed during this study are included in its supplementary information files.

Funding/Grant support: None

Disclosure of Potential Conflicts of Interest: All authors do not have any financial support related to this article. 
Ethics Approval: All procedures in this study were in accordance with the ethical standards of the responsible committee on human experimentation (institutional and national) and with the Helsinki Declaration of 1964 and later versions. Informed consent was obtained from each patient. All patient data sets were collected after approval by the institutional review board of Tsukuba University Hospital (Approval No. R01-030).

Consent to participate: Informed consent was obtained from the patient.

Consent for publication: Informed consent was obtained from the patient.

\section{Author contributions:}

Osamu Shimomura: Project development, Data Collection, Formal analysis, Resources, Manuscript Writing. Tatsuya Oda: Project development, Investigation, Manuscript Writing, Supervision. Manami Doi: Formal analysis, Data Collection, Resources. Yoshihiro Miyazaki: Writing- Reviewing and Editing. Kazuhiro Takahashi: Writing- Reviewing and Editing. Kinji Furuya: Writing- Reviewing and Editing. Jaejeong Kim: Writing- Reviewing and Figure visualization. Shoko Moue: Writing- Reviewing and Editing. Yohei Owada: Writing- Reviewing and Editing. Koichi Ogawa: Writing- Reviewing and Editing. Yusuke Ohara: Writing- Reviewing and Editing. Yoshimasa Akashi: Writing- Reviewing and Editing. Tsuyoshi Enomoto: Writing- Reviewing and Editing. Shinji Hashimoto: Study concept, Investigation, Formal analysis, Data Collection, Resources.

\section{Acknowledgements:}

The authors thank the surgical team staff, especially Dr. Toshiki Sakamoto of Tsukuba Memorial Hospital, Tsukuba-city, Japan. We also wish to thank Prof. Katashi Fukao and Prof. Nobuhiro Ohkochi, the former chair of the Department of Surgery, University of Tsukuba, Japan, for their useful discussions and valuable support.

\section{References}

1. Grobmyer SR, Rivadeneira DE, Goodman CA, Mackrell P, Lieberman MD, Daly JM: Pancreatic anastomotic failure after pancreaticoduodenectomy. Am J Surg. 2000 Aug; 180(2): 117-20.

2. Kimura W, Miyata H, Gotoh M, Hirai I, Kenjo A, Kitagawa $Y$ et al. A pancreaticoduodenectomy risk model derived from 8575 cases from a national single-race population (Japanese) using a webbased data entry system: the 30-day and in-hospital mortality rates for pancreaticoduodenectomy. Ann Surg. 2014 Apr; 259(4): 773-80.

3. Ghaferi AA, Birkmeyer JD, Dimick JB. Variation in hospital mortality associated with inpatient surgery. N Engl J Med. 2009 Oct 1; 361(14): 1368-75.

4. Kawai M, Kondo S, Yamaue H, Wada K, Sano K, Motoi F et al. Predictive risk factors for clinically relevant pancreatic fistula analyzed in 1,239 patients with pancreaticoduodenectomy: multicenter 
data collection as a project study of pancreatic surgery by the Japanese Society of Hepato-BiliaryPancreatic Surgery. J Hepatobiliary Pancreat Sci. 2011 Jul; 18(4): 601-8.

5. Grobmyer SR, Kooby D, Blumgart LH, Hochwald SN. Novel pancreaticojejunostomy with a low rate of anastomotic failure-related complications. J Am Coll Surg. 2010 Jan; 210(1): 54-9.

6. Fujii T, Sugimoto H, Yamada S, Kanda M, Suenaga M, Takami H et al. Modified Blumgart anastomosis for pancreaticojejunostomy: technical improvement in matched historical control study. J Gastrointest Surg. 2014 Jun; 18(6): 1108-15.

7. Oda T, Hashimoto S, Miyamoto R, Shimomura O, Fukunaga K, Kohno K et al. The Tight Adaptation at Pancreatic Anastomosis Without Parenchymal Laceration: An Institutional Experience in Introducing and Modifying the New Procedure. World J Surg. 2015 Aug; 39(8): 2014-22.

8. Kojima T, Niguma T, Watanabe N, Sakata T, Mimura T. Modified Blumgart anastomosis with the "complete packing method" reduces the incidence of pancreatic fistula and complications after resection of the head of the pancreas. Am J Surg. 2018 Nov; 216(5): 941-8.

9. Hirono S, Kawai M, Okada Kl, Miyazawa M, Kitahata Y, Hayami S et al. Modified Blumgart Mattress Suture Versus Conventional Interrupted Suture in Pancreaticojejunostomy During Pancreaticoduodenectomy: Randomized Controlled Trial. Ann Surg. 2019 Feb; 269(2): 243-51.

10. Sugimoto M, Takahashi S, Kojima M, Kobayashi T, Gotohda N, Konishi M. In Patients with a Soft Pancreas, a Thick Parenchyma, a Small Duct, and Fatty Infiltration Are Significant Risks for Pancreatic Fistula After Pancreaticoduodenectomy. J Gastrointest Surg. 2017 May; 21(5): 846-54.

11. Howard TJ, Rhodes GJ, Selzer DJ, Sherman S, Fogel E, Lehman GA. Roux-en-Y internal drainage is the best surgical option to treat patients with disconnected duct syndrome after severe acute pancreatitis. Surgery. 2001 Oct; 130(4): 714-9.

12. Vasquez CR, Chestovich P, Muthusamy VR, Donahue TR. Resolution of persistent pancreaticopleural fistula after an internal drainage procedure. Am Surg. 2014 Jan; 80(1): E19-21.

13. Bassi C, Marchegiani G, Dervenis C, Sarr M, Abu Hilal M, Adham M et al. The 2016 update of the International Study Group (ISGPS) definition and grading of postoperative pancreatic fistula: 11 Years After. Surgery. 2017 Mar; 161(3): 584-91.

14. Bassi C, Dervenis C, Butturini G, Fingerhut A, Yeo C, Izbicki J et al. Postoperative pancreatic fistula: An international study group (ISGPF) definition. Surgery. 2005 Jul; 138(1): 8-13.

15. Oda T, Hashimoto S, Shimomura O, Yamada K, Ohara Y, Fukunaga K et al. Inter-Anastomosis Drainage Tube Between the Pancreas and Jejunum: A Novel Technique for Preventing Pancreatic Fistula after Pancreaticoduodenectomy. J Am Coll Surg. 2015 Sep; 221(3): e55-60.

16. Kawakatsu S, Inoue Y, Mise Y, Ishizawa T, Ito H, Takahashi Y et al. Comparison of pancreatojejunostomy techniques in patients with a soft pancreas: Kakita anastomosis and Blumgart anastomosis. BMC Surg. 2018 Oct; 18(1): 88.

\section{Tables}


Tables are available in the Supplemental Files section.

\section{Figures}

Figure.1

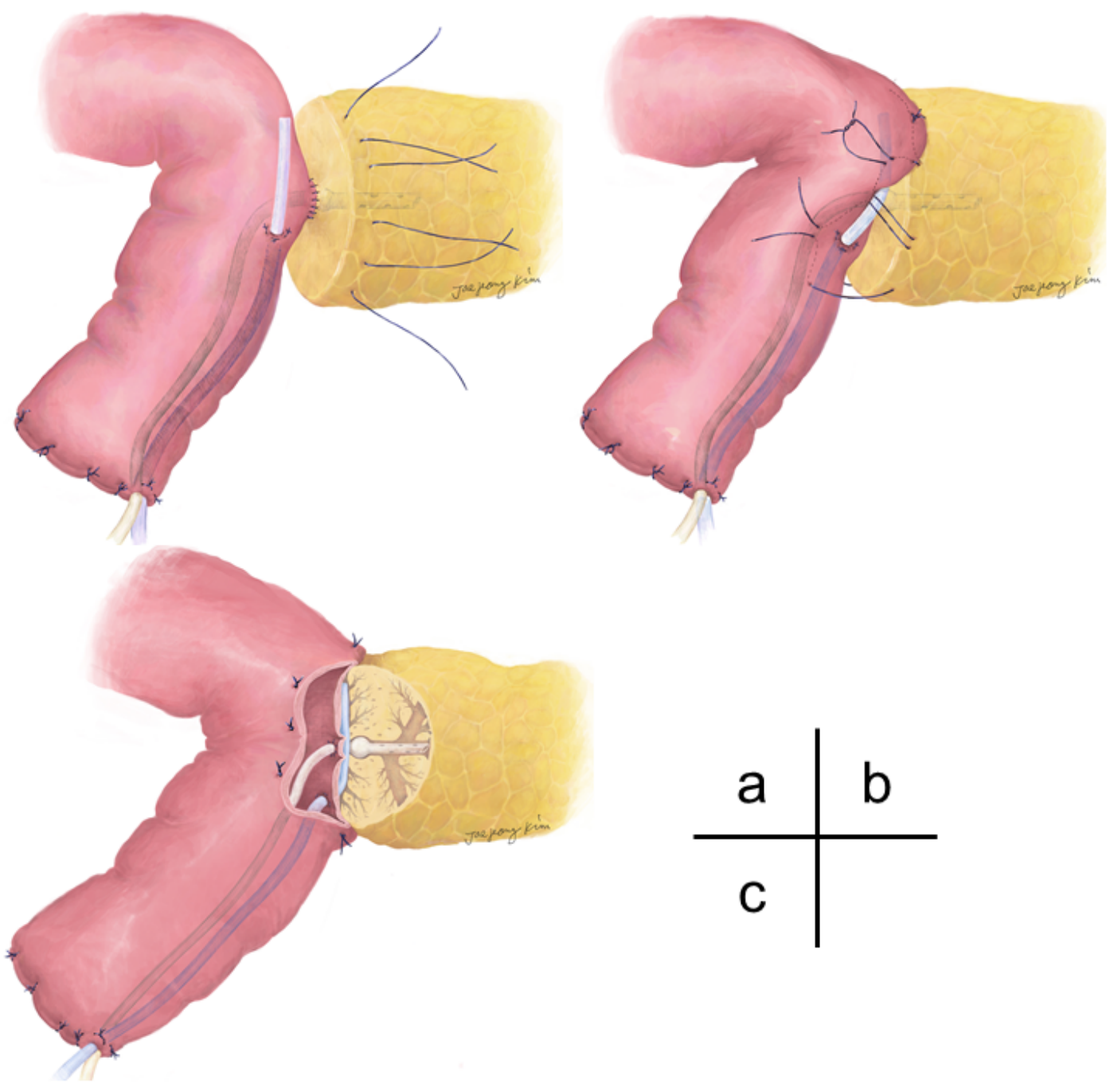

Figure 1

The placement of the inter-anastomosis drainage (IAD) tube (Figure 1a-c): 
After completing the duct-to-mucosa anastomosis, the IAD tube was placed at the interspace between the jejunal wall and pancreatic parenchyma through the end of the jejunum (a). A $10 \mathrm{Fr}$ silicone tube (BLAKE Silicone Drains-Hubless, Ethicon) was arranged in an "I" shape and was inserted into the interanastomosis space. The external drainage tube of the MPD stent was placed and fixed, and Blumgart mattress sutures (3-0 PDS- $\mathbb{Z}$, Ethicon) were ligated by wrapping the IAD tube inside (b). The IAD tube was completely enveloped by the jejunal wall and pancreatic parenchyma (c). Reinforcing 4-0 PDS-II sutures were occasionally added to both edges in order to ensure a watertight seal. At the end of the surgery, the IAD suction tube was connected to a low-pressure continuous-suction device. 
Figure.2

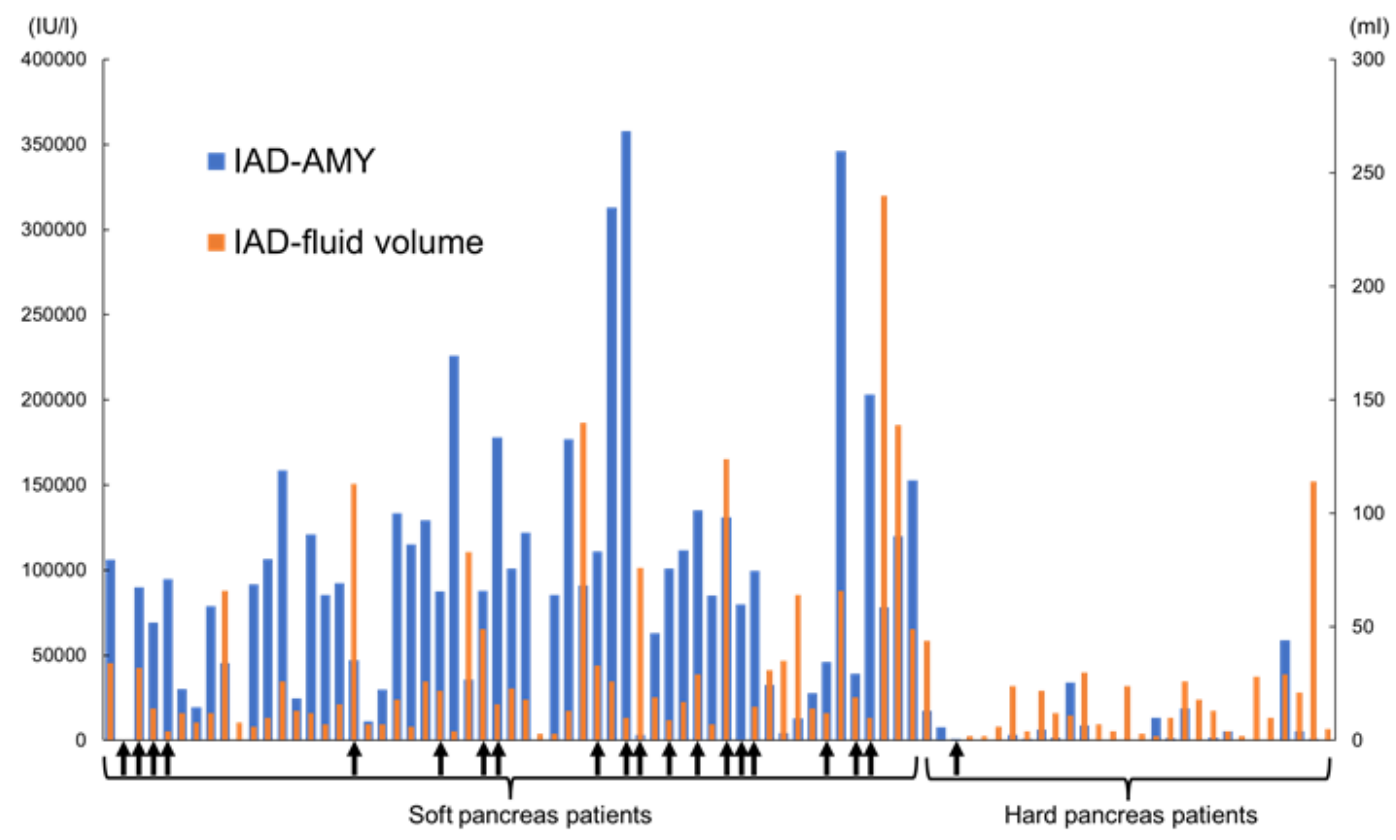

Figure 2

Amylase value and the Drainage volume of each patient in IAD+B group. Bar-graph (left side) indicates the amylase value of IAD tube collection, and the right side indicates the volume of the IAD collection. Arrows revealed the existence of grade B or C pancreatic fistula. 


\section{Figure.2}

a.

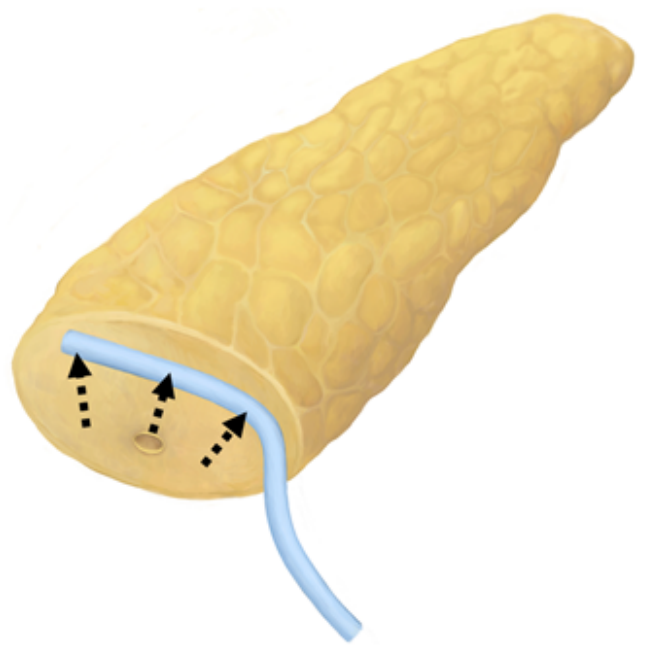

b.

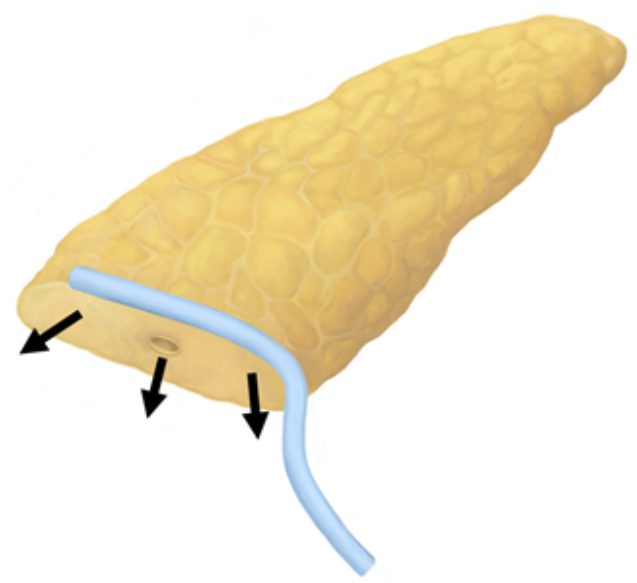

c.

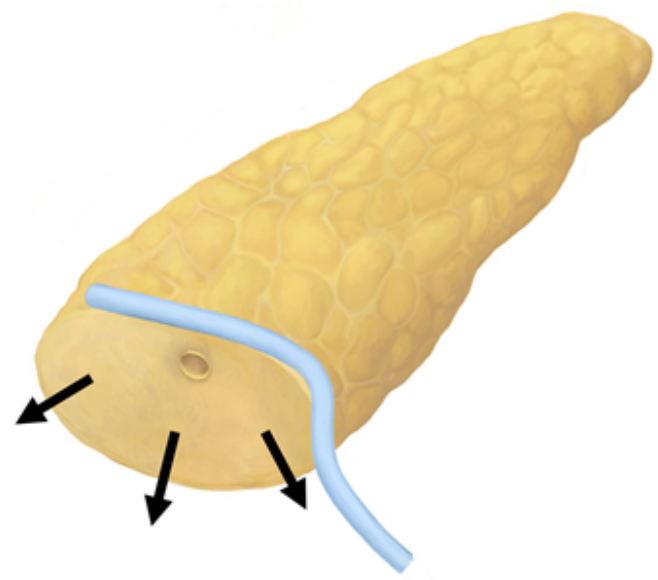

\section{Figure 3}

The inter-anastomosis drainage (IAD) in Blumgart type pancreatojejunostomy works effectively when the pancreas resection surface is wide (not small) and the main pancreatic duct locates on the dorsal side because IAD tube placed fine in water-tight manner (a.). IAD tube placement did not work well when the resection surface is thin or small (b.) and the main pancreatic duct locate on the ventral side (c.). 


\section{Supplementary Files}

This is a list of supplementary files associated with this preprint. Click to download.

- table1.tif

- table2.tif

- table3.tif

- Supplementarylawdata.xlsx 